\title{
Skin Metastasis in Breast Cancer Patients; a Case Series
}

\author{
Sara Rehman, Muhammad Atif Naveed \\ Department of Radiology, Shaukat Khanum Memorial Cancer Hospital and Research Centre, Lahore, \\ Pakistan
}

Received: 04 November 2019/Accepted: 05 December 2019

\section{G open ACCess}

Correspondence: Dr. Sara Rehman, Department of Radiology, Shaukat Khanum Memorial Cancer Hospital and Research Centre, Lahore, Pakistan. Email: sararehman_88@ yahoo.com

Citation: Rehman $S$ and Naveed MA. Skin metastasis in breast cancer patients; a case series. J Cancer Allied Spec [Internet]. 2020 Jan.6; 6(1):e1002957.https://doi. org/10.37029/jcas.v6i1.307

Copyright: (C) 2020 Rehman and Naveed. This is an open access article distributed under the terms of the Creative Commons Attribution License, which permits unrestricted use, distribution, and reproduction in any medium, provided the original author and source are credited.

Funding: Nill.

Competing interests: Nill.

\begin{abstract}
Introduction: Breast cancer is the most common malignancy in women. It frequently metastasizes to bones, lungs and liver. Although rare, skin metastasis may also take place. It may also be the presenting feature of initial or recurrent breast cancer. The assessment of recurrent metastatic disease involving skin after mastectomy can be challenging because of the benign-appearing clinical presentation. The purpose of this case series was to explore the clinical and radiological presentation of skin metastasis in patients of breast cancer. Materials and Methods: This is a retrospective case series of breast cancer patients with skin lesions on chest and abdomen at the time of initial presentation, or post-treatment such as, after mastectomy or breast conservation therapy; who underwent various radiological investigations including mammography, ultrasound scan, computed tomography (CT) scan and magnetic resonance imaging from 1 May 2018 to 30 September 2019 at Shaukat Khanum Memorial Cancer Hospital and Research Centre, Pakistan. Results: A total of eight cases were identified, out of which seven were females. The most common presentation consisted of the presence of metastatic nodules which were seen in $62.5 \%$ (five out of eight) of the patients. Other features consisted of erythematous or plaque-like skin thickening on clinical examination, increased density with indistinct margins seen on a mammogram and diffuse oedematous changes in the skin with small irregular mass or infiltration into subcutaneous tissues were visualised on ultrasound and CT studies. Conclusion: Skin metastasis from breast cancer most commonly presents as nodules, although rarely they may present as plaques or diffuse skin thickening. Awareness of diverse manifestations of skin metastasis is of utmost importance in early diagnosis and management.
\end{abstract}

Key words: Breast cancer, case series, cutaneous lesions, radiological imaging, skin metastasis

\section{Introduction}

Skin metastasis from primary visceral malignancies is an uncommon presentation. ${ }^{[1]}$ The overall incidence of skin metastasis is $5.3 \% .{ }^{[2]}$ Skin metastasis is more frequently seen in breast cancer than in any other visceral malignancy in women. ${ }^{[1]}$ The incidence of breast carcinoma cutaneous manifestation is $23.9 \% .^{[1]}$ The most common sites of breast carcinoma skin manifestation are the chest wall, 
abdomen and back, but they can occur at the extremities and in the head/neck region. ${ }^{[3]}$

Skin lesions can be the first sign for the initial occurrence of the breast cancer. They have a wide range of clinical manifestations, ranging from painless single or multiple hard nodules to induration and oedema of the skin of the breast, known as the orange-peel appearance (peau d'orange). ${ }^{[3]}$ Peau d'orange of breast is considered to be a common sign for inflammatory breast cancer (IBC). However, it may present in various other benign conditions as well, such as cellulitis or lymphedema. ${ }^{[3,4]}$ IBC is an aggressive form of breast cancer. It often has systemic manifestations and a poor prognosis. Therefore, early diagnosis and prompt intervention are crucial for management. ${ }^{[4]}$ Conversely, skin lesions can be one of the initial signs for the recurrence of breast cancer. ${ }^{[5]}$ This is considered clinically to be a diagnostic challenge because clinical signs of skin metastasis share close similarity to the skin changes that take place after antineoplastic therapy (surgery or radiation therapy). ${ }^{[5]}$ To improve the understanding of skin lesions associated with breast cancer, the present study was conducted to explore the clinical and radiological presentations of skin metastasis in patients of breast cancer.

\section{Materials and Methods}

A retrospective electronic medical chart review was conducted of breast cancer patients with skin lesions on the chest and abdomen at the time of initial presentation, or post-treatment such as, after mastectomy or breast conservation therapy, who underwent various radiological investigations such as mammography, ultrasound, computed tomography (CT) scan, or magnetic resonance imaging (MRI) from 1 May 2018 to 30 September 2019 at Shaukat Khanum Memorial Cancer Hospital and Research Centre, Pakistan. Breast cancer patients without skin lesions were excluded from the study. This study was approved by the Institutional Review Board of Shaukat Khanum Memorial Cancer Hospital and Research Centre (03-07-19-01).
During the hospital visit, patients underwent a comprehensive clinical assessment comprising of a complete history, physical examination, radiological imaging, interventional procedures and pathological analysis. For radiological assessment, all patients underwent ultrasound examination of breasts or mastectomy scar site using 7.5-10 MHz linear array transducer Toshiba Aplio XG, Toshiba Xario 100 and Toshiba Aplio 500. In addition, where indicated, mammography was performed on dedicated mammographic equipment, consisting of Senographe Essential (GE) and Mammomat Inspiration (Siemens), acquiring standard mediolateral oblique and craniocaudal views of the breasts. Likewise, computerised tomography (CT) scan of chest, abdomen and pelvis with contrast was conducted using Prime SP Aquilion Scanner (160 slices). For contrast, $1.5-1.67 \mathrm{ml} / \mathrm{kg}$ of iodinated intravenous contrast medium was injected at a rate of $1.5-2 \mathrm{ml} / \mathrm{s}$ by a Medrad-Salient Power Injector, with a scan delay of $70 \mathrm{~s}$ after the onset of injection. Typical imaging parameters were $120 \mathrm{kVp}, 150$ $\mathrm{mAs}$ and $5 \mathrm{~mm}$ slice thickness with a pitch of 1.2. Similarly, dynamic contrast-enhanced MRI study of breasts was performed using Ingenia 1.5-T (Philips) with a dedicated breast surface coil, noncontrast axial T1, STIR and post-contrast dynamic enhanced axial and sagittal T1 Fat Sat images were acquired after IV administration of contrast medium $(0.1 \mathrm{mmol} / \mathrm{kg}$ gadovist). The diagnosis was made based on findings of cytology/histopathology of ultrasound-guided fine-needle aspiration (FNA)/ core biopsy.

Staging of the tumour was based on clinical examination, cytology/histopathology and radiological findings (as per American Joint Committee on Cancer [AJCC] TNM $7^{\text {th }}$ edition classification) ${ }^{[6]}$

\section{Results}

\section{Case 1}

A 68-year-old woman presented with left breast diffuse skin thickening for 2 years. On clinical examination, there was a diffuse firm mass extending 
from the inframammary fold to left hypochondrium, left flank and epigastric region, which was inseparable from the overlying erythematous skin.

She underwent a mammogram and an ultrasound of the left breast. Her mammogram showed an indistinct increased density mass in the lower central aspect in the left breast [Figure 1.1 $a$ and b]. On ultrasound, there was diffuse skin thickening with infiltration into subcutaneous tissues in the left breast involving the inframammary fold extending into the anterior abdominal wall [Figure 1.2]. Following the scans, the patients had an ultrasoundguided biopsy from the subcutaneous mass and wedge biopsy of the skin. These were positive for invasive lobular carcinoma (ILC) - Grade II. Furthermore, wedge biopsy histopathological sample suggested that tumour was extending into the dermis. The patients also underwent CT chest, abdomen and pelvis study with contrast. It showed extensive soft-tissue thickening in the left breast, inframammary fold extending along the length of the anterior abdominal wall, involving the rectus sheath and the anterior abdominal muscles, with significant overlying skin thickening and subcutaneous oedema. The underlying peritoneal lining was also thickened, with fat stranding in the greater omentum and neovascularity in the anterior abdominal wall. Fat stranding was noted in the left axilla as well, with soft tissue involving the serratus anterior, left shoulder girdle muscles and latissimus dorsi muscle [Figure 1.3a and b].

Based on the medical history, clinicohistopathological analysis and radiological findings, the multidisciplinary team (MDT) decided that the patients should be started on hormonal therapy (letrozole $2.5 \mathrm{mg}$ once a day). The patients did not come back to the hospital for follow-up.

\section{Case 2}

A 36-year-old woman presented with the right breast lump for 6 months. Her clinical examination was significant for a fixed $3 \mathrm{~cm}$ lump at 6 o'clock and multiple enlarged right axillary lymph nodes.
Her mammogram showed an increased density mass in the right lower central breast and her right breast ultrasound showed an irregular hypoechoic infiltrative mass in the right breast at 5 o'clock. In addition, there was a hypoechoic dermal nodule present at 6 o'clock, measuring $7 \mathrm{~mm}$ at a distance of $4 \mathrm{~mm}$ from the main mass [Figure 2.1]. The right axilla showed multiple enlarged lymph nodes and an irregular mass measuring $31 \mathrm{~mm} \times 26 \mathrm{~mm}$.
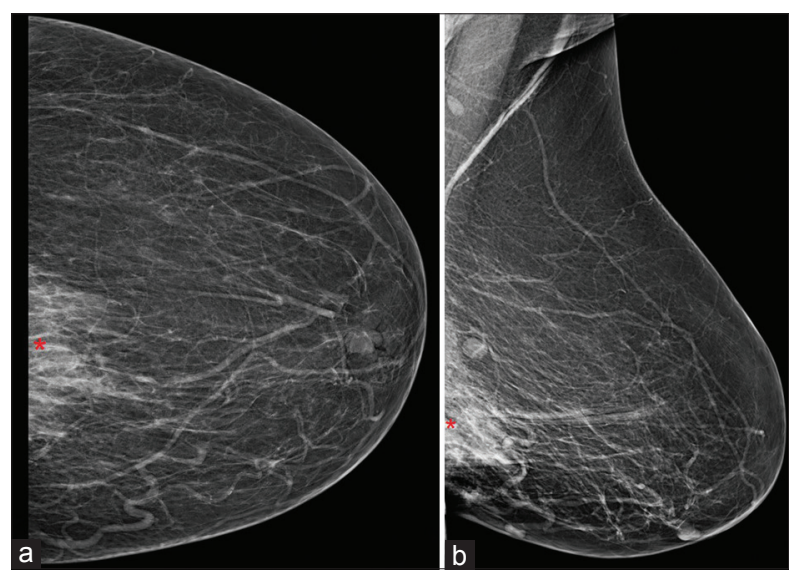

Figure 1.1: ( $a$ and $b$ ): Craniocaudal (a) and mediolateral oblique (b) views of left breast show increased density mass with indistinct margins (shown as red*) in the lower central aspect. No grouped suspicious microcalcifications are visualised

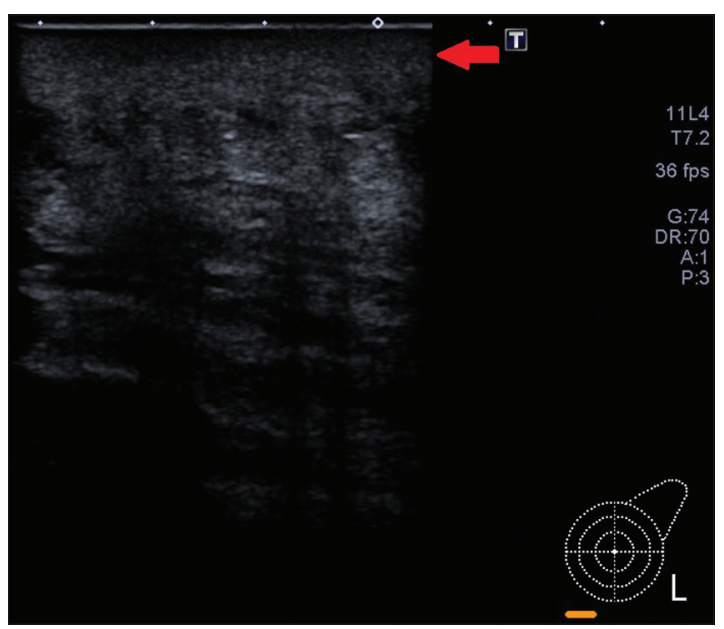

Figure 1.2: Greyscale images of the left breast are demonstrating diffuse skin thickening with infiltration into subcutaneous tissues (red arrow) in the left breast involving the inframammary fold extending into the anterior abdominal wall 
Ultrasound-guided core biopsies were performed for the right breast 5 o'clock and the right axillary masses. The histopathological analyses showed the presence of invasive ductal carcinoma (IDC) Grade III in both masses. Furthermore, the patients underwent ultrasound-guided FNA in the right axillary lymph node and right breast 6 o'clock skin nodule. FNA of the right lymph node was positive for metastatic carcinoma, and FNA for breast nodule was significant for mammary carcinoma. CT chest, abdomen and pelvis with contrast were performed for the metastatic workup. It was notable for the

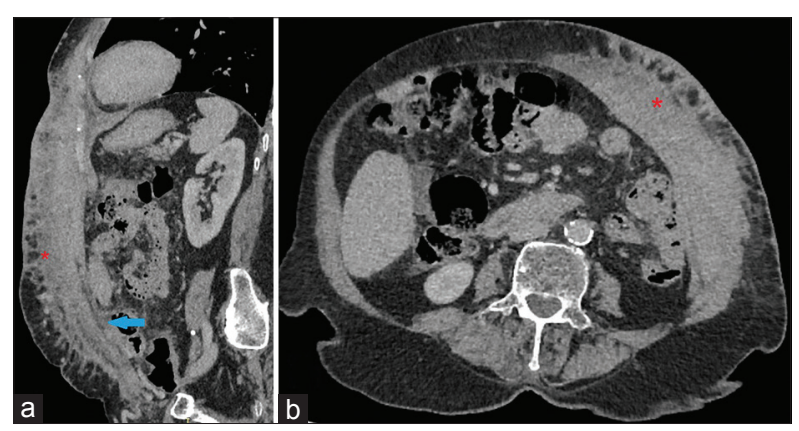

Figure 1.3: ( $a$ and b): Sagittal (a) and axial (b) images of contrast-enhanced CT through chest, abdomen and pelvis in soft-tissue window show extensive soft-tissue thickening in the left breast along the inframammary fold extending along the length of the anterior abdominal wall, involving the rectus sheath and the anterior abdominal muscles (shown as red*). The underlying peritoneal lining was also thickened, with fat stranding in the anterior omentum (blue arrow)

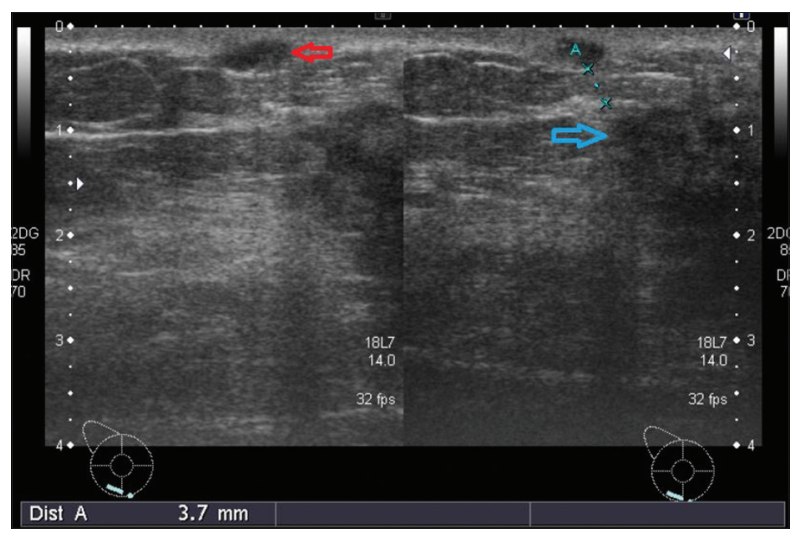

Figure 2.1: Greyscale ultrasound images of the right breast show a hypoechoic dermal nodule (red arrow) at 6 o'clock, measuring $7 \mathrm{~mm}$ at $4 \mathrm{~mm}$ from the main mass (blue arrow) presence of a right breast mass with ipsilateral axillary lymphadenopathy. A small skin nodule was also noted at 6 o'clock [Figure 2.2]. There was no evidence for distant metastatic disease.

The MDT agreed to start the patients on neoadjuvant chemotherapy followed by right mastectomy. Breast conservation was not considered as a treatment option due to the presence of metastatic skin nodule which upstaged the disease to T4b/ Stage III.

\section{Case 3}

A 58-year-old man presented with the right breast lump for 5 years with blood-stained nipple discharge. Clinical examination was significant for a $4 \mathrm{~cm}$ right breast retro-areolar lump with ulceration of the overlying skin and nipple. In addition, another nodular $1 \mathrm{~cm}$ mass was palpable. This was $2 \mathrm{~cm}$ medial to the main mass.

Mammogram of this patients showed a high-density spiculated mass in the retroareolar region in the right breast with overlying skin tethering, thickening and nipple retraction. A circumscribed high-density mass was also seen in the upper outer quadrant. Furthermore, a suspicious high-density irregular mass was seen near the axillary tail [Figure 3.1 a and b] and few small lymph nodes were noted in the right

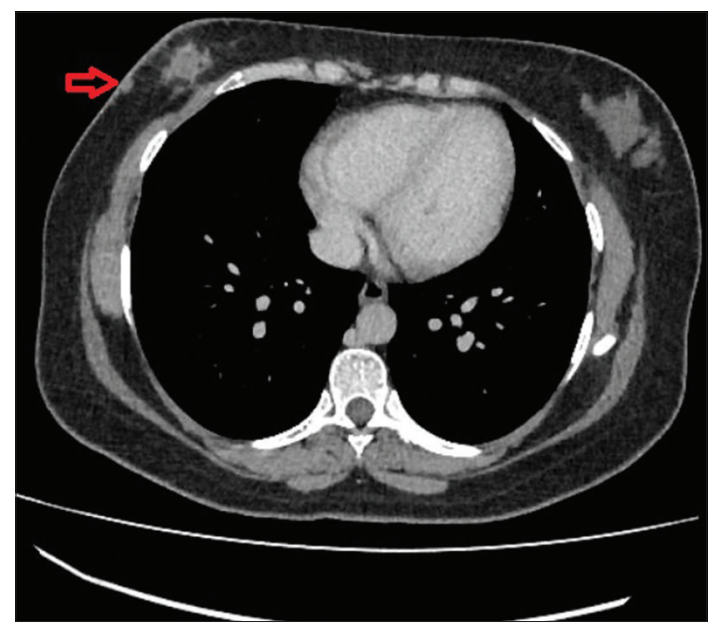

Figure 2.2: Soft-tissue window of the axial image of contrast-enhanced CT through chest showing small skin nodule (red arrow) at 6 o'clock 
axilla. Correlative ultrasound scan of breast showed an irregular hypoechoic mass in the right breast retroareolar region with the surrounding area of architectural distortion. It was inseparable from the overlying skin. There was a hyperechoic nodule in the skin/superficial tissues, measuring $9 \mathrm{~mm}$ at 2 o'clock, at a distance of $35 \mathrm{~mm}$ from the main mass [Figure 3.2]. Another irregular hypoechoic mass was observed at 9 o'clock, at a distance of $35 \mathrm{~mm}$

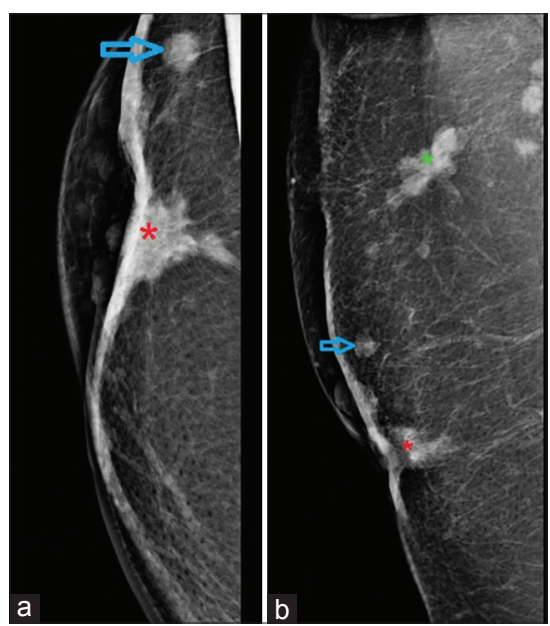

Figure 3.1: ( $a$ and $b$ ): Craniocaudal ( $a$ ) and mediolateral oblique (b) views of right breast showing a high-density spiculated mass (shown as red*) in the retroareolar region right breast with overlying skin tethering, thickening and nipple retraction. A circumscribed high-density mass is also seen in the upper outer quadrant (blue arrow). Suspicious high-density irregular mass is also visualised near the axillary tail (shown by green*)

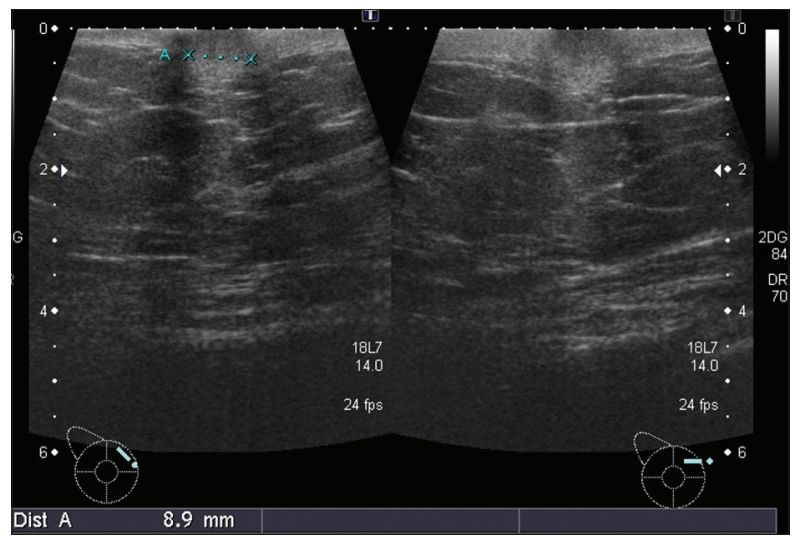

Figure 3.2: Greyscale ultrasound image of the right breast showing a hyperechoic nodule in the skin measuring $9 \mathrm{~mm}$ at 2 o'clock from the main mass. Few adjacently placed and similar-appearing hypoechoic masses were noted near the axillary tail. Ultrasound-guided biopsy was performed for the right breast retroareolar main mass and 9 o'clock and axillary tail satellite masses. The histopathological analysis showed IDC - Grade II in both of the breast masses, and the lymph node in axillary tail mass was significant for metastatic carcinoma. Ultrasound-guided FNA from the right breast skin mass at 2 o'clock revealed mammary carcinoma on cytopathology.

CT chest and abdomen with contrast showed right breast retroareolar mass with adjacent $10 \mathrm{~mm}$ softtissue nodule and ipsilaterally enlarged axillary lymph nodes along with widespread metastatic lung disease (Figure 3.3a and b). Nuclear medicine bone scintigraphy study showed increased radiotracer uptake involving the right sixth rib near the costovertebral junction with underlying sclerotic change. This was concerning for osseous metastasis.

The MDT recommended the patients to undergo systemic chemotherapy, followed by a CT scan to reassess the extent and severity of the disease. A CT chest and abdomen were performed after 6 months of completing systemic chemotherapy. It showed partial interval treatment response according to RECIST (Response Evaluation Criteria in Solid Tumours) criteria. ${ }^{7]}$

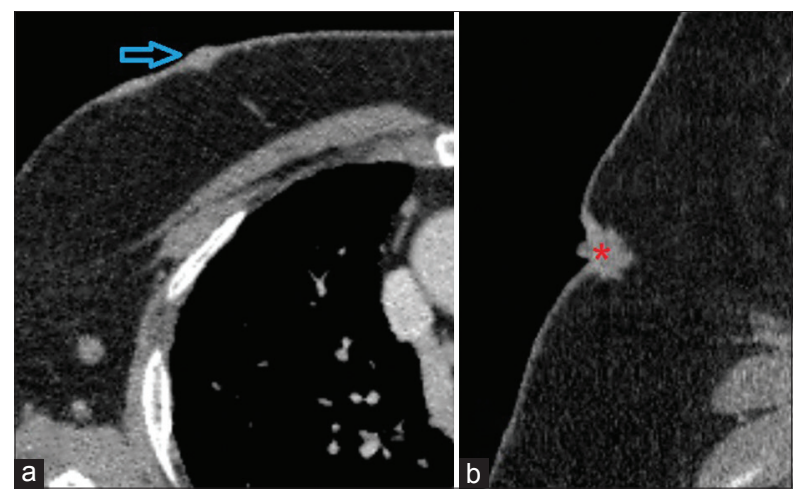

Figure 3.3: ( $a$ and $b$ ): Axial (a) and sagittal (b) images of soft-tissue window of contrast-enhanced CT chest showing right breast 02:00 skin nodule (blue arrow) and retroareolar spiculated mass (shown as red*) with overlying nipple and areolar complex retraction 


\section{Case 4}

A 54-year-old female presented with history of right breast lump for 2 months. Her examination was significant for the presence of a $3 \mathrm{~cm}$ lump with overlying skin involvement at 12 o'clock, and multiple palpable right axillary lymph nodes.

She underwent bilateral mammographic studies with standard views. On the right side, there was a spiculated mass in the upper outer quadrant of the breast with overlying skin tethering. Another spiculated mass was seen in the upper outer quadrant in the posterior aspect. The right axillary region was clear. On the left side, the breast showed two spiculated masses in the upper inner quadrant [Figure 4.1 a-d].

Her ultrasound scan of breasts showed an irregular hypoechoic spiculated mass in the right breast at a 12 o'clock, measuring $18 \mathrm{~mm} \times 22 \mathrm{~mm}$, which appeared inseparable from overlying skin. An irregular hyperechoic dermal nodule, measuring $9 \mathrm{~mm}$ was seen at a 10 o'clock, at a distance of $6 \mathrm{~mm}$ from the 12 o'clock mass [Figure 4.2a and b]. The right axillary region had multiple enlarged suspicious lymph nodes. The left breast showed two small hypoechoic masses at 10 o'clock and 9 o'clock, measuring $5 \mathrm{~mm}$ and $4 \mathrm{~mm}$ each.

The patients underwent ultrasound-guided biopsy of the bilateral breast masses. These were positive for Grade II-ILC. In addition, the patients completed ultrasound-guided FNA for the right breast dermal nodule and the right axillary lymph node. The cytopathology analyses of these lesions were significant for the presence of malignant cells in the right breast dermal nodule and metastatic carcinoma in the right axillary lymph node.

The MDT recommendations for this patients were to complete neoadjuvant chemotherapy followed by a right mastectomy, and left breast wire-guided localised excision of the two masses.

\section{Case 5}

A 63-year-old woman presented with palpable nodules along the left chest wall on the previous surgery scar site along with palpable right axillary lymph nodes. She had been treated with breast conservation therapy for the left breast IDC Grade III 5 years ago. She had a local recurrence 2 years ago, for which neoadjuvant
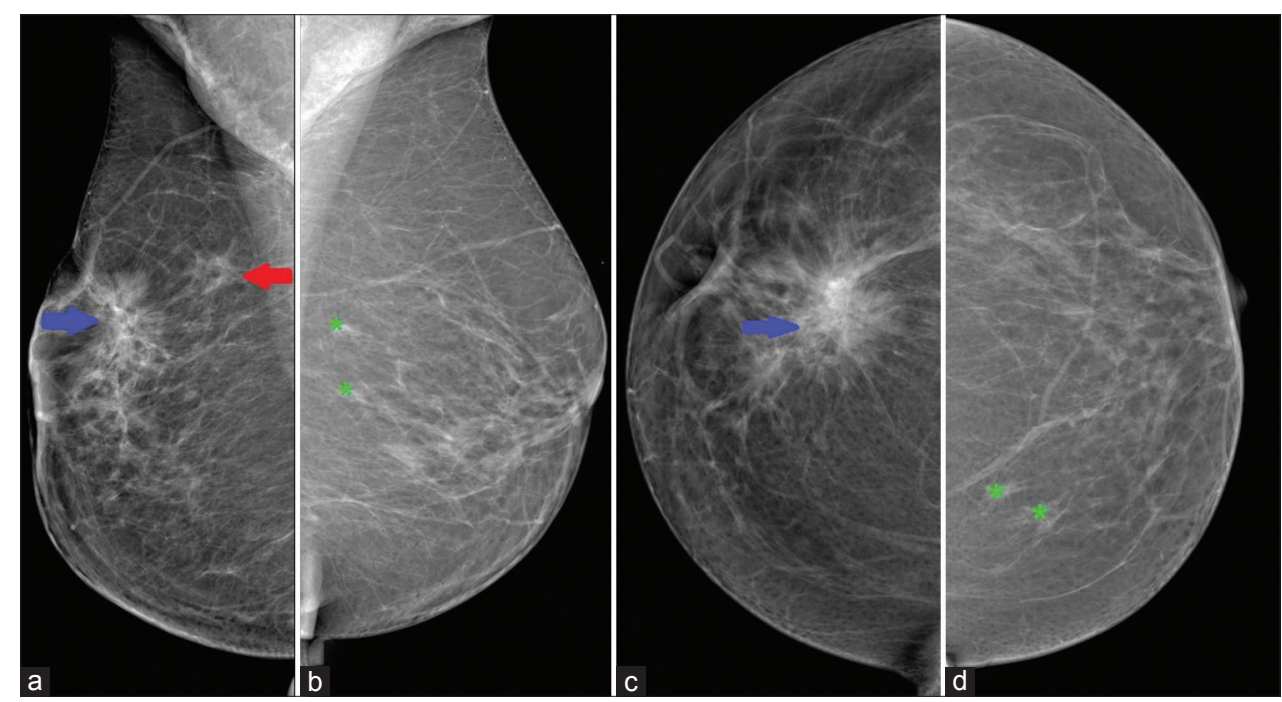

Figure 4.1: (a-d): Mediolateral oblique (MLO) (a) and craniocaudal (CC) (c) views of the right breast show a spiculated mass (blue arrow) in the upper outer quadrant of the right breast with overlying skin tethering. Another spiculated mass (red arrow) is also seen in the upper aspect on MLO view in the posterior aspect. MLO (b) and CC (d) views of the left breast show two spiculated masses (shown by green*) in the upper inner quadrant 

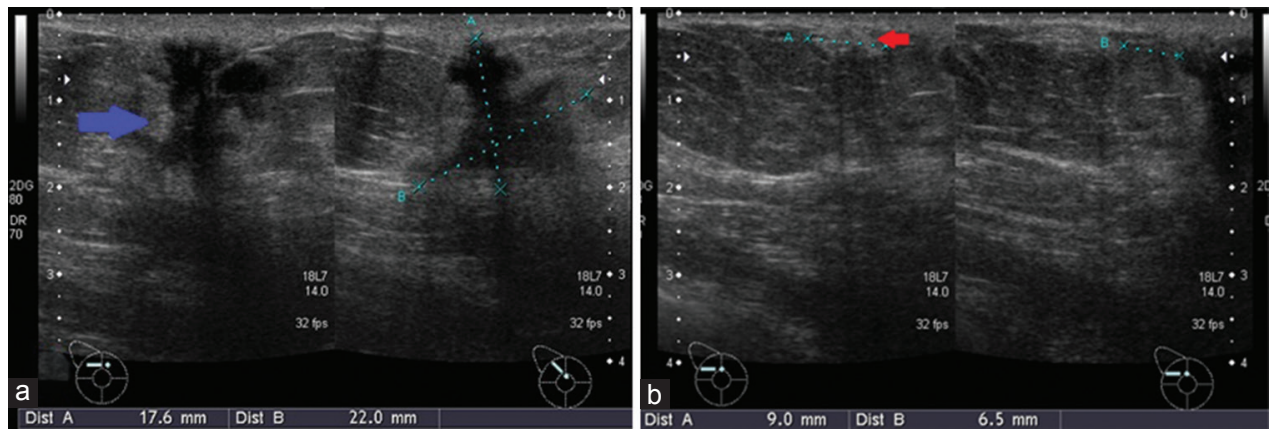

Figure 4.2: (a): Greyscale ultrasound images showing (a) irregular hypoechoic spiculated mass in the right breast 12 o'clock (blue arrow), which appears inseparable from overlying skin, (b) an irregular hyperechoic dermal nodule (red arrow), seen at 10 o'clock in the right breast

chemotherapy was administered followed by left breast mastectomy.

Her mammogram of the right breast showed skin thickening with enlarged right axillary lymph nodes [Figure 5.1]. She had a dynamic breast MRI scan of the breasts, which suggested small enhancing masses with diffusion restriction, along the mastectomy scar and an enlarged contralateral right axillary lymph node [Figure 5.2a-d]. MRI scan was followed by an ultrasound-guided FNA from left mastectomy scar site nodules and a core biopsy of the right axillary lymph node and results were positive for the presence of malignant cells and metastatic carcinoma, respectively.

The MDT advised systemic chemotherapy for this patients. However, the patients was unable to complete the course of therapy due to multiple systemic side effects. She was placed on exemestane (25 mg once a day). Her follow-up ultrasound breast and CT of chest, abdomen and pelvis showed regression of disease (as per RECIST criteria $\left.{ }^{[7]}\right)$ and there was no evidence of metastasis.

\section{Case 6}

A 35-year-old woman presented with fever, left chest wall skin thickening and erythema extending into the right breast and left flank for 2 months after completion of radiotherapy to the left chest wall. She had a history of left breast multifocal IDC Grade III, for which neoadjuvant chemotherapy was administered and modified radical mastectomy

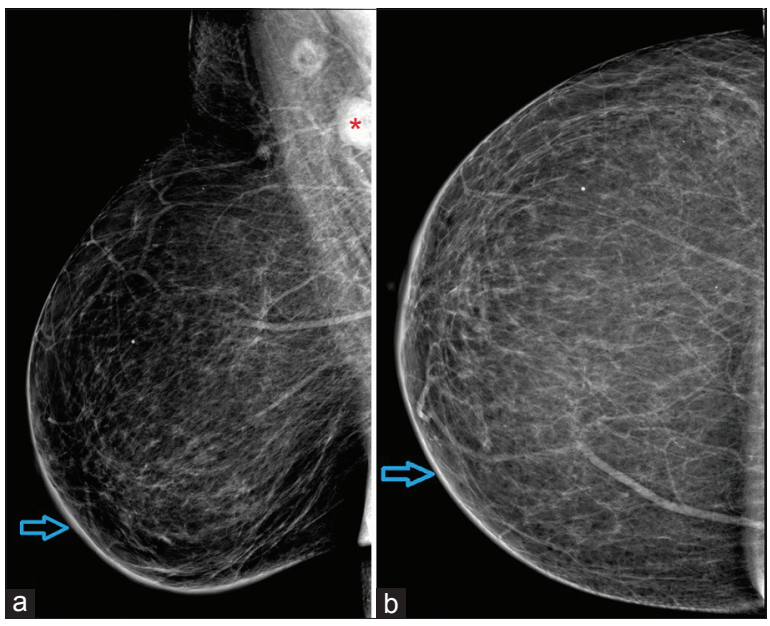

Figure 5.1: ( $a$ and b): (a) Mediolateral oblique and (b) craniocaudal views of the right breast showing interval development of the right breast skin thickening (blue arrow) and enlarged right axillary lymph nodes (shown by red*)

(MRM) was performed subsequently. Radiotherapy was also completed after the surgery. Initially, the aforementioned presenting symptoms and clinical findings were considered as post-radiation inflammatory changes and she was given antiinflammatory medication; however, after no significant improvement in symptoms over corresponding weeks, the patients underwent a series of testing to rule out local recurrence of the disease.

Ultrasound of the left chest wall showed diffuse oedematous changes involving the skin and subcutaneous tissues along with the mastectomy 


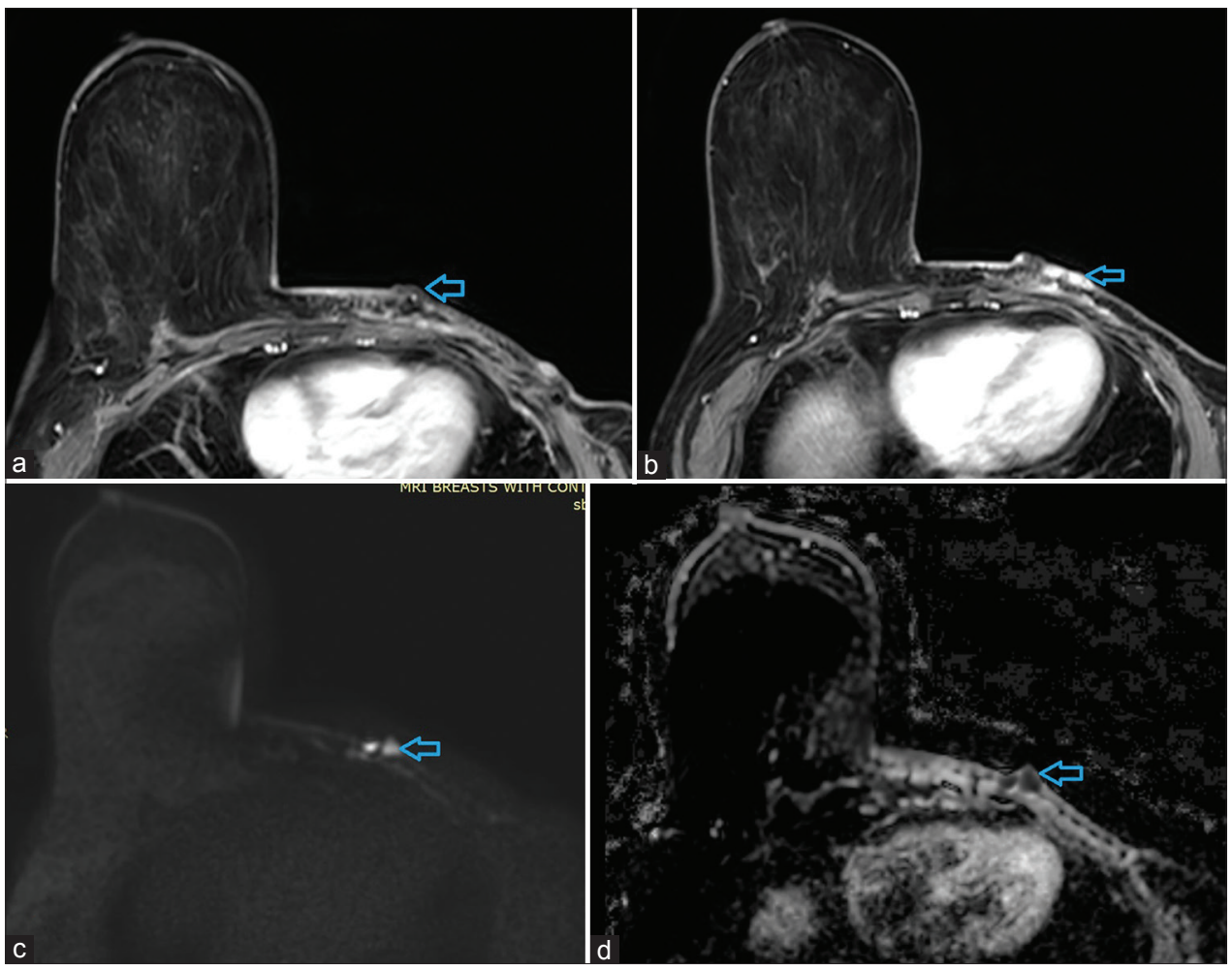

Figure 5.2: ( $a-d)$ : ( $a$ and b) Dynamic contrast enhanced magnetic resonance imaging (MRI) breasts showing enhancing nodules (blue arrow) along left mastectomy scar site, with evidence of restricted diffusion, (c) same lesion in a diffusionweighted MRI sequence and (d) apparent diffusion coefficient sequence

site along with the extension to the left lateral chest wall. Irregular masses, measuring $9 \mathrm{~mm}$ in maximum thickness, were seen along with the scar site and left axilla [Figure 6.1a]. Furthermore, the enlargement of contralateral right axillary lymph nodes was appreciated. An ultrasoundguided biopsy [Figure 6.1 b] was performed for the irregular mass along with the mastectomy scar site. It was positive for IDC - Grade III. An ultrasoundguided FNA was performed for the enlarged right axillary lymph node as well as from the irregular mass in the left axilla, and cytopathological analyses suggested the presence of metastatic carcinoma.

CT chest abdomen and pelvis with contrast were performed and it showed indistinct enhancement/thickening along the left mastectomy scar site/anterior chest wall and subcutaneous nodular thickening along the left lateral chest wall [Figure 6.2a and b]. In addition, there were bilateral pleural effusions, with patchy multifocal ground-glass opacities and interstitial septal thickening associated with mediastinal and hilar lymphadenopathy.

Based on the immediate radiological and pathological findings, MDT recommended the patients to be started on systemic chemotherapy. Follow-up imaging has not yet been performed.

\section{Case 7}

A 54-year-old female presented with a small $1.5 \mathrm{~cm}$ skin based ulcerated mass at the medial end of the infra-mammary fold. She had a history of lumpectomy done for the right breast IDC Grade II, followed by chemotherapy and radiotherapy 14 years ago.

Bilateral breast mammogram was performed with standard views which showed post-surgical architectural distortion in the lower central aspect 

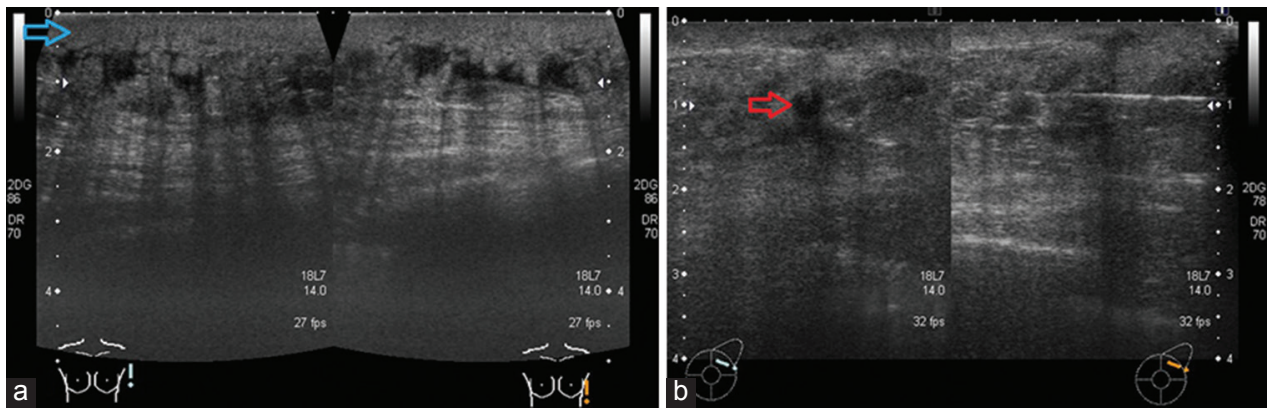

Figure 6.1: ( $a$ and b): (a) Greyscale ultrasound images of right mastectomy scar site showing diffuse oedematous changes involving the skin and subcutaneous tissues (blue arrow) along with the mastectomy site along with the extension to the left lateral chest wall. (b) Irregular mass (red arrow) along with the scar site and ultrasound-guided biopsy of this mass
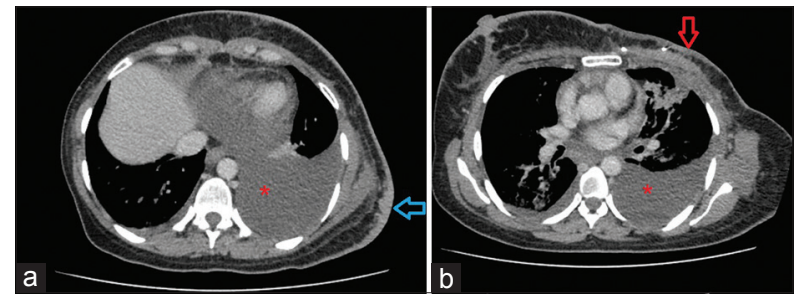

Figure 6.2: ( $a$ and b): Axial slices of contrast-enhanced CT scan through the chest and upper abdomen soft-tissue window showing indistinct enhancement/thickening along with the left modified radical mastectomy scar site/anterior chest wall (red arrow) and subcutaneous nodular thickening along left lateral chest wall (blue arrow). Bilateral pleural effusions (red*) also seen

(skin based nodule was not covered due to its location) [Figure 7.1a and b]. Ultrasound scan of breasts showed a circumscribed hypoechoic mass at 05:00 position near the inframammary fold, in the dermal planes measuring about $16 \mathrm{~mm} \times 6 \mathrm{~mm}$ and extending into the subcutaneous tissues. An enlarged intramammary lymph node was seen near the axillary tail [Figure 7.2a and b]. The patients underwent ultrasound-guided FNA of the dermal nodule and the intramammary lymph node. The cytopathological analyses were significant for mammary carcinoma and reactive lymphoid hyperplasia, respectively.

CT chest, abdomen and pelvis with contrast were performed that showed changes of lumpectomy and a heterogeneously enhancing small soft-tissue mass involving the lower inner quadrant of the right breast with overlying skin infiltration. The right axilla

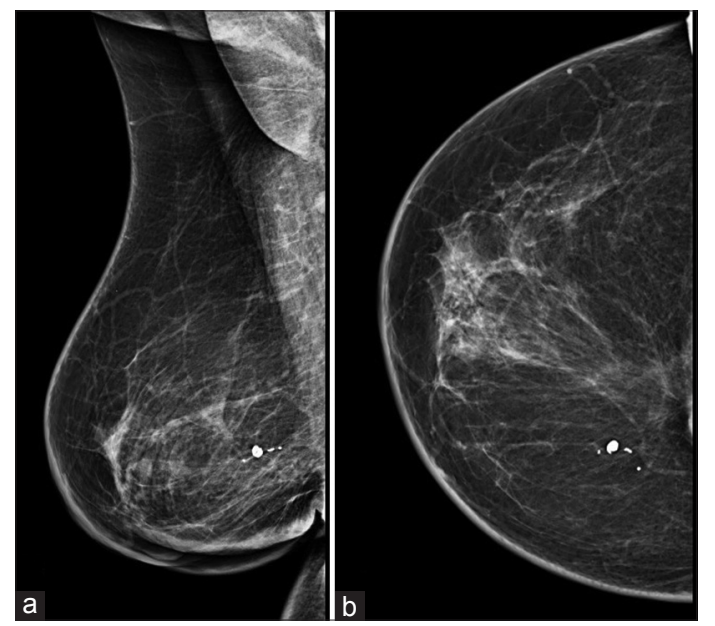

Figure 7.1: ( $a$ and $b$ ): (a) Mediolateral oblique and (b) craniocaudal views of the right breast, showing post-surgical architectural distortion in the lower central aspect and mild skin thickening (skin based nodule not covered due to its location)

showed changes of axillary lymph node dissection with no abnormal soft-tissue mass noted near the axillary scar site. Furthermore, there was a large right-sided pleural effusion along with extensive heterogeneously enhancing pleural based masses, concerning for metastatic disease [Figure 7.3a]. Enlarged nodal masses were noted in the right internal mammary station, pretracheal and right paratracheal region [Figure 7.3b].

The MDT decision was to start the patients on systemic chemotherapy. Follow-up imaging has not yet been performed. 

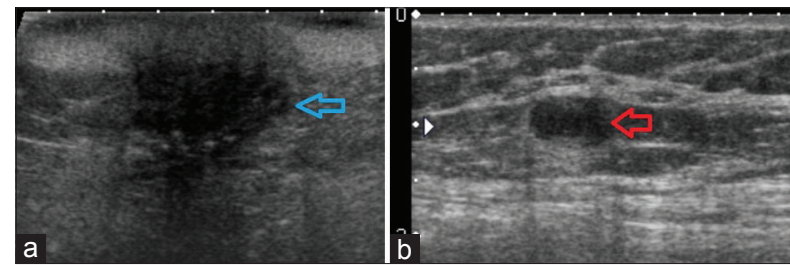

Figure 7.2: ( $a$ and b): (a) Greyscale ultrasound image shows a circumscribed hypoechoic mass (blue arrow) at 5 o'clock near the inframammary fold, in the dermal planes, extending into the subcutaneous tissues. (b) Enlarged intramammary lymph node (red arrow) also seen near the axillary tail
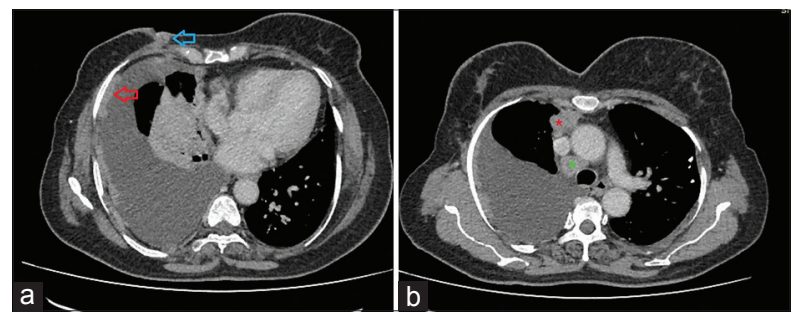

Figure 7.3: ( $a$ and b): (a) Contrast-enhanced CT through chest axial section soft-tissue window shows a small soft tissue mass (blue arrow) involving the lower inner quadrant of the right breast with overlying skin infiltration. Large right-sided pleural effusion along with the extensive heterogeneously enhancing pleural based masses (red arrow) is also noted. (b) Enlarged nodal mass is seen in the right internal mammary station (shown by red $^{*}$ ) as well as in the paratracheal and right paratracheal region (shown by green*)

\section{Case 8}

A 50-year-old female presented with rapid onset of rash on her left anterior chest, upper left arm and left axilla. She had a 1 day history of blister formation on the dorsal aspect of left-hand finger with high-grade fever. She had a history of left breast IDC Grade II and was treated with neoadjuvant chemotherapy followed by left MRM and radiation therapy 2 years ago. On examination, there was erythema on the left anterior chest, left arm and left axilla. Clinical assessment was that this was either an allergic reaction or cellulitis.

Ultrasound showed localised collection with thick walls without any echogenic foci or septations or abnormal vascularity under the lateral end of left mastectomy scar representing post-surgical

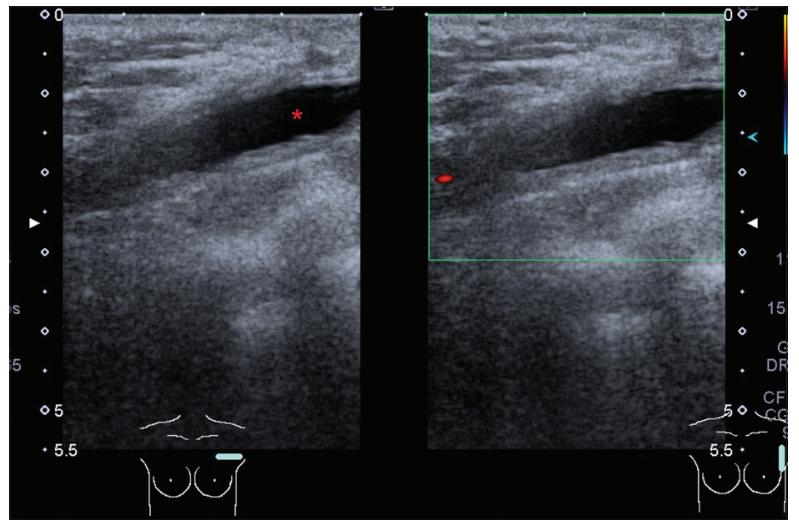

Figure 8: Greyscale ultrasound images show localised collection with thick walls without any echogenic foci or septations under the lateral end of left mastectomy scar representing seroma (shown by red*)

seroma [Figure 8]. Ipsilateral arm and axilla showed diffuse oedematous changes involving the skin and subcutaneous tissues. No radiologically suspicious axillary lymph nodes were seen. These findings were suggestive of cellulitis, and the patients was treated with systemic antibiotics for 2 weeks. Arm physiotherapy and exercises were also advised.

Patients reported to follow-up in the outpatient clinic after 2 weeks and was found to be asymptomatic with the resolution of redness and swelling at the scar site.

\section{Discussion}

Breast cancer is the most common primary malignancy that presents with cutaneous metastasis. ${ }^{[1]}$ Cutaneous metastasis is usually a sign of advanced breast cancer; rarely, it may be the first sign of undiagnosed breast cancer..$^{[1]}$ Moreover, patients may present years after diagnosis and treatment have been completed..$^{[5]}$ These skin lesions have variable clinical appearances and can be mistaken for benign skin lesions.

In a retrospective review by Mordenti et al., 164 cases of skin metastasis from breast carcinoma were examined to determine the most common clinical and histopathological presentations. ${ }^{[9]}$ Skin papules and/or nodules were the most common manifestation found in 
$80 \%$ of patients, telangiectatic carcinomas were seen in $11 \%$, erysipeloid carcinomas in $3 \%$, 'en cuirasse' carcinomas in 3\%, alopecia neoplastica in $2 \%$ and a zosteriform type in $0.8 \%{ }^{[9]}$ In our case series, metastatic nodules were the most common presentation of $\mathrm{CM}$, which was seen in five out of eight patients.

In the initial diagnostic workup of breast cancer patients, evidence of metastatic skin nodules or inflammatory carcinoma is very important while considering the surgical management of the disease. The presence of metastatic dermal nodules, in the absence of distant metastasis, is considered as the local advancement of the disease. It is staged as $T 4 \mathrm{~b}$, as per AJCC TNM $7^{\text {th }}$ edition classification. ${ }^{[6]}$ Regardless of the tumour size and involvement of the regional lymph nodes, the presence of skin involvement places any tumour into Stage III and it is considered a contraindication to breast conservation therapy. ${ }^{[4]}$ Studies of neoadjuvant chemotherapy for breast conservation still typically exclude cases with cutaneous metastasis; similarly, some studies narrate that breast conservation is contraindicated when skin involvement exists in tumours of any size. ${ }^{[4]}$

There were two cases that presented with extensive diffuse skin thickening and erythema clinically resembling carcinoma erysipeloides or carcinoma en cuirasse. Our first case had skin lesions at the time of the initial presentation, while the sixth case was a treated breast cancer patients. Carcinoma erysipeloides (CE) is an uncommon but distinctive form of cutaneous metastasis that usually manifests clinically as a fixed erythematous patch or plaque resembling cellulitis or erysipelas. Al Ameer et al. reported a case of CE with underlying breast carcinoma, which was initially being treated as eczematous dermatitis for 2 months. ${ }^{[10]}$ CE has rapid clinical onset and aggressive progression that requires prompt diagnosis and early management to improve survival. However, it often presents as a diagnostic challenge as it may be easily mistaken for other entities; these include mastitis, cellulitis, erysipelas, thrombophlebitis, venous congestion, allergic reactions, postsurgical lymphedema or hematoma, post-radiotherapy dermatitis, herpes zoster infection, primary squamous, or basal cell carcinoma. ${ }^{[11]}$ Carcinoma en cuirasse is an unusual skin metastasis of breast cancer with diffuse carcinomatous cutaneous and subcutaneous infiltration that may affect the chest and abdomen, by extension. ${ }^{[8]}$ Reich et al. have reported a case of carcinoma en cuirasse as an initial clinical manifestation of breast cancer in a woman with 15-month history of asymptomatic, previously untreated erythematous and indurated skin lesions of the right chest. ${ }^{[12]}$ Our eighth case had a similar clinical presentation of diffuse erythematous skin thickening involving mastectomy scar site, but imaging features were not favouring malignancy. This patients was followed up until she had complete resolution of symptoms, which occurred after a course of antibiotic treatment.

Diagnosis of cutaneous metastasis is very important in breast cancer patients for correct staging as well as a possibility of metastatic disease in patients presenting with cutaneous lesions over breast mimicking benign dermatological conditions. Disease relapse in post-surgery and/ or post-radiotherapy breast cancer patients may be confused with post-treatment cellulitis or inflammation. The imaging modalities, including mammography, ultrasound scan, CT and MRI aid in the detection of skin metastasis and in excluding possible benign causes. If there is any uncertainty regarding the aetiology of the cutaneous lesion, or no resolution with initial treatment, the threshold for a tissue diagnosis should be low for accurate diagnosis and treatment.

\section{References}

1. Navaratnam AV, Chandrasekharan S. Remote cutaneous breast carcinoma metastasis mimicking dermatitis. Indian J Dermatol 2015;60:106.

2. Krathen RA, Orengo IF, Rosen T. Cutaneous metastasis: A meta-analysis of data. South Med J 2003; 96:164-7.

3. Helm TN, Lee TC. Dermatologic Manifestations of Metastatic Carcinomas Clinical Presentation, Medscape; 2019.

4. Chen AM, Meric-Bernstam F, Hunt KK, Thames HD, Outlaw ED, Strom EA, et al. Breast conservation after 
neoadjuvant chemotherapy. Cancer 2005; 103: 689-95.

5. Virmani NC, Sharma YK, Panicker NK, Dash KN, Patvekar MA, Deo KS. Zosteriform skin metastases: Clue to an undiagnosed breast cancer. Indian J Dermatol 2011;56:726-7.

6. Edge SB. AJCC Cancer Staging Manual. $7^{\text {th }}$ ed. New York: Springer, American Joint Committee on Cancer; 2010.

7. Nishino M, Jagannathan JP, Ramaiya NH, Van den Abbeele AD. Revised RECIST guideline version 1.1: What oncologists want to know and what radiologists need to know. AJR Am J Roentgenol 2010;195:281-9.

8. Oliveira GM, Zachetti DB, Barros HR, Tiengo A, Romiti N. Breast carcinoma en Cuirasse--case report. An Bras Dermatol 2013;88:608-10.

9. Mordenti C, Peris K, Fargnoli MC, Cerroni L, Chimenti S. Cutaneous metastatic breast carcinoma. Acta Dermatovenerol 2000;9:143-8.

10. Al Ameer A, Imran M, Kaliyadan F, Chopra R.
Carcinoma erysipeloides as a presenting feature of breast carcinoma: A case report and brief review of literature. Indian Dermatol Online J 2015;6:396-8.

11. Chow HT, Tran K, Millar EK, Lynch J, Murrell DF. Diverse presentations of carcinoma erysipelatoides from a teaching hospital in Australia. Case Rep Dermatol Med 2012;2012:134938.

12. Reich A, Samotij D, Szczęch J, Woźniak Z, Szepietowski J. Carcinoma en cuirasse as an initial manifestation of inflammatory breast cancer. Postepy Dermatol Alergol 2016;33:142-5.

\section{Authorship Contributions}

Conceived and designed the analysis: SR; Collected the data: SR; Contributed data or analysis tools: SR and MAN; Performed the analysis: N/A; Wrote the paper: SR and MAN. 